\title{
Transition pathways of e-mobility services
}

\author{
E. Gould ${ }^{1,2}$, W. Wehrmeyer ${ }^{1} \&$ M. Leach ${ }^{1}$ \\ ${ }^{1}$ Centre for Environmental Strategy, University of Surrey, UK \\ ${ }^{2}$ Schneider Electric, $U K$
}

\begin{abstract}
The concept of mobility is developing in to a complete solution composed of various modes of transport and alternative ownership models. Evidence suggests that Mobility-as-a-Service will become integral to society, incorporating mobile apps for payment and location-aided services, thus ensuring ease of use and functionality [1]. This is a considerable opportunity to decarbonise transport within cities, reducing the need for private car ownership and utilising electric vehicles within the mobility model. There is however uncertainty of what and how this should be implemented and therefore requires further research within the transitions field.

This research will investigate city mobility services, specifically e-mobility. This will be considered in the context of product-service systems to explore the existing market and identify transition pathways. Use-oriented services are the primary focus as the business models are most explicitly linked to car sharing, renting and pooling.

The level of uptake of e-mobility services is reliant upon the interest and acceptance of society and the mechanisms put in place by Government and private enterprise. An expected outcome of this research is a requirement for greater collaboration between Government and private enterprise, in order to initially fund city schemes but also ensure they are viable in the long term. It can be expected that data must be shared to a greater extent between the public and private sector and that this is accessible to citizens. Both of these factors will affect people's choice of transport mode through availability of vehicles and real time information on travel options.
\end{abstract}

Keywords: electric vehicles, urban, e-mobility, mobility-as-a-service, product service systems, use-oriented. 


\section{Introduction}

Cars currently contribute $12 \%$ of total EU emissions of carbon dioxide; the European Union target requires a $40 \%$ reduction by 2021 [2]. As the fastest growing contributor to climate change methods of transportation need to be challenged [3]. Broadening the availability of alternatives to car ownership has the opportunity to reduce transport emissions. The concept of mobility has dramatically changed over recent years; evidence suggests that Mobility-as-aService (MaaS) will become integral to society. MaaS incorporates mobile apps for payment and location-aided services to ensure ease of use and functionality [1]. A transition to MaaS will require a fundamental change in market concepts in order to "shift from one socio-technical system to another i.e. a system innovation" [4].

A study conducted by LSE [5] indicated that shifting to greener modes of transport is the most important strategy to achieve sustainable transport. MaaS can introduce alternatively fuelled vehicles (AFVs) in to the city transport portfolio. This paper will specifically consider electric vehicles (EVs) in an urban context. EVs being used across a city at multiple vehicle pick-up and drop-off locations, removes many of the barriers to EVs such as high purchase price and range anxiety. More so, the niche application of EVs within this context can assist the transition to an established and broader EV network.

The move from a product-led business model of traditional car ownership and leasing, to a product-service system introduces car sharing, renting and pooling. This introduces new ownership and revenue structures that use subscription or pay-as-you-go based models, transferring vehicle responsibility and risk on to the service provider [6]. This transition in vehicle use requires a shift in behaviour and asset culture to dematerialise the transport sector. In seeking to deepen understanding on how to achieve this within a city, the paper will explore examples of e-mobility models. Product-service systems will be analysed with particular focus on use-orientated services, as the business models are most explicitly linked to car sharing, renting and pooling. It will then be discussed how cities can appropriately transition to such use-oriented services.

\section{Product-service systems}

Sustainability of the automobile industry requires behavioural and system-level changes [7]. In order to achieve this there is an emphasis to adopt a product-service system (PSS). This has been defined as "a marketable set of products and services capable of jointly fulfilling a user's need" [8]. Mont [9] further explains PSS as "a system of products, services, supporting networks and infrastructure that is designed to be: competitive, satisfy customer needs and have a lower environmental impact than traditional business models".

Product-service systems have the opportunity to continuously innovate and develop new offers [9]. In the product-service segment of the automotive industry the most adaptable and innovative products are the additional services such as the assistance services and location-aided apps. These are particularly important in 
mobility solutions and add value through customer experience, building unique relationships and customer loyalty [10].

Broadly PSSs can be classified in to three main categories: (i) productorientated services; (ii) use-oriented services; and (iii) result-orientated services. This paper will focus on use-orientated services, by which the products remain central to the offer and are managed by a service provider, whilst the utility is provided as an output service unit for the desired level of use e.g. mobile phone contract [9, 11]. In doing so alternative profitable revenue streams can be identified and considerable changes in behaviour and culture can be achieved [11].

Applying PSS to e-mobility services, there are three approaches that can be introduced: (i) the sale of the use of the product instead of the product itself, (ii) the change to a leasing society, and (iii) the change in consumer attitudes from sales to service orientation [9]. These three PSS elements will be considered in regards to the examples given in Section 3 and then discussed in Section 4.

In order to evaluate PSS initiatives, Ehrenfeld [12] introduced five key evaluative criteria: (i) evidence of 'higher-order' learning amongst stakeholders, (ii) changes in infrastructure and institutional practice, (iii) changes in vehicle design, manufacture and end-of-life management, (iv) changes in vehicle ownership structure, and (v) changes in modes of producer-user interactions. Although all five criteria are necessary to introducing and managing PSSs, e-mobility services relates directly to (iv) and (v) categories. Furthermore, Ehrenfeld indicates that changing the product concept eases the system transition; therefore it could be argued that using EVs in car clubs instead of internal combustion vehicles will encourage drivers to adopt the service [11]. This will be discussed further in Sector 4.

\section{Use-oriented services}

Use-oriented mobility services increase efficiency of private transport in cities, for example "one vehicle can service around 15 times more users daily than a privately owned vehicle" [6]. As seen in Figure 1, there are three main use-orientated services within the automotive industry. These can be used independently to each other and other forms of transport, or can be in used in conjunction, with a multitude of mobility services.

\subsection{Product lease}

Vehicle leasing is growing in popularity both by companies and private users as a means to finance vehicle purchase. This model is used by corporations as a means to lower their overhead costs, by providing employees leased cars rather than long term company cars. There are two forms of leasing (i) traditional use and return, and (ii) eco-leasing whereby the product is returned, dissembled and re-used as raw materials. Eco-leasing introduces a more sustainable approach to car ownership than outright purchase. 


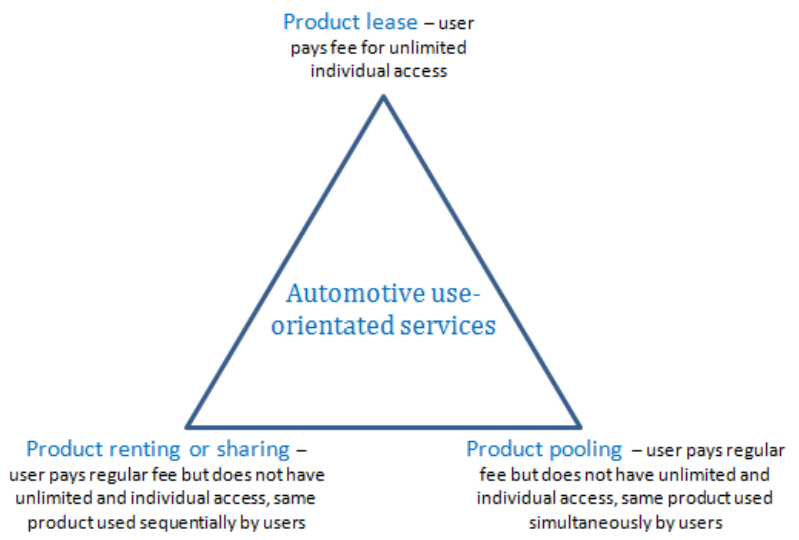

Figure 1: Types of use-orientated services within the automotive industry.

Product leasing could be argued to be an intermediary phase between ownership and vehicle sharing. It shifts behaviour from owning assets to a pay-asyou-go model, whilst having unlimited and individual access.

A mobility offer from $\mathrm{BMWi} 360^{\circ}$ offers a range of services including 'mobility solutions' for those who purchase a BMWi EV. Customers wishing to carry out extended journeys beyond the range of an EV can hire an appropriate vehicle [13]. This is an encompassing approach to transport as it encourages 'green' behaviour where possible but the convenience of distance, speed and/or size for specific journeys.

\subsection{Product renting or sharing}

There are a growing number of vehicle rental or sharing schemes that offer access to a variety of vehicles to suit customer needs as required. In such instances the provider will retain ownership of the vehicle and is often responsible for maintenance and repair; the user pays a regular fee but does not have unlimited and individual access. There are a number of examples of vehicle renting or sharing across the globe that account for 1,788,000 car sharing members that have access to 43,500 cars [14]. This is the biggest opportunity for e-mobility services to leverage, below are three examples of EV schemes in progressive European cities:

\subsubsection{AutoLib', Paris}

Following trials Autolib' launched in June 2012 with 250 of their own 'Bluecars' and 250 stations and centres. Today there are approximately 2,000 'Bluecars' and 4,300 stations within the forty square mile city $[15,16]$. Autolib' was the first public service EV plan to be orchestrated in a European city and is suggested to have substituted 22,500 privately owned vehicles, the equivalent of over a hundred million miles per year in an internal combustion engine (ICE) [15].

There are a range of payment models that offer flexibility and freedom to travel across the city using a point-to-point business model unlocking the full potential 
of electric car sharing [16]. Autolib' supplies the vehicles and returns "the city's investment with subscription revenue and a parking space leasing agreement", it was expected this would take seven years but due to high utilisation rates the City's investment will be covered in less than four years [8]. The city is known for parking bay shortages, Autolib' provides access to 4,300 parking spaces (and charging points) in desirable locations provided by the City of Paris. Furthermore, "cars in Paris are driven only about $5 \%$ of the time and stay parked the remaining $95 \%$ of the time" thus enforcing the business case of e-mobility [6].

Autolib' has normalised EVs in Paris and dramatically altered the transportation mix within the city. The visibility of the vehicles, cost effectiveness and ease of use are all key elements that challenge the embedded technological lock-in of ICE vehicles. The high utilisation rates of Autolib' demonstrates that drivers are prepared to use AFVs for city travel and that despite a well-established public transport network in Paris, car travel remains desirable and/or a more appropriate transport mode for certain journeys. It is reported that the majority of Autolib' users are $25-49,80 \%$ are men and the average distance is less than six miles [17]. This would suggest that the importance of owning a vehicle is diminishing in younger generations and the shift from product models to service models may increase in popularity and viability.

\subsubsection{Source London, London}

In 2014 the Ballore Group took ownership of 1,300+ charging points across London from the previous Government led 'Plugged in Places' scheme. Charging infrastructure will increase to 4,500 by 2018 , this will be a considerable shift for London, especially if a pay-as-you-go model is used. The scheme will aim to be high volume, high frequency with reservations holding for thirty minutes encouraging it to be used on an ad hoc basis to meet both spontaneous and planned journeys. With $37 \%$ of all trips in London per day (26 million trips a day) made by private transport, the opportunity for EVs to populate the city is vast [18].

Gaining parking bays on a leasing agreement from 32 Boroughs will be challenging for Source London as each Borough has its own parking requirements and legislation. Car2Go, a car sharing scheme that operated in London ceased operations due to the difficulty of working with the Boroughs. This will require careful consideration for Source London as an integrated approach across the city will be required for a viable scheme, alternatively it will be a more local offer for those Boroughs involved.

\subsubsection{DriveNow, Berlin}

DriveNow is BMW's car sharing service which operates in five German cities as well as San Francisco and most recently London. In Berlin there are eight BMW models available for hire including the pure electric, Active E. The scheme has various DriveNow packages that specify payment either per minute or per hour depending on the customers demand. Berlin has 900 cars in and around the S-Bahn ring, twelve of which are pure electric. The service team refuel/recharge the vehicles but if the tank/battery is low resulting in the driver 'filling up', twenty minutes are granted to the customer's account. This incentive encourages drivers 
to interact with the vehicle, that being more important for the Active E to educate and familiarise drivers with the technology, thus unlocking path dependencies.

DriveNow has streamlined the process of activating vehicles, charging and parking encouraging adoption and replication for other cities. It is reported that $6 \%$ of 155,000 members globally did not purchase a new car directly because of DriveNow and that $16 \%$ of members deferred buying a new car [19]. This indicates the impact car clubs can have upon a city's congestion through reducing road vehicles alongside assisting to reinstate the balance of emissions and air quality.

\subsection{Product pooling}

Vehicle pooling is similar to that of vehicle renting but the car is used simultaneously by the user rather than sequentially. This requires considerably less investment than car leasing/sharing with the need for fewer vehicles [10]. There are two distinct client bases, the public and members often in a workplace environment.

\subsubsection{Hertz BilPool, Oslo}

Hertz operate a car pooling service across Norway, with Oslo being the primary area with a high concentration of vehicles including the Nissan Leaf and Volvo C30 electric. There are two different levels of membership based on hours or kilometres with optional weekend deals. It is an A-A model requiring the car to be returned to the same location after use [20]. This does not provide the same level of flexibility for drivers but guarantees parking availability and charging infrastructure.

Hertz BilPool also offers corporate membership providing companies with access to vehicles without requiring their own fleet. This removes the need for company cars and/or a leasing agreement removing asset management, maintenance and high overhead costs. Companies using this model can gradually introduce AFVs to employees, bearing little risk.

For a population that is aligned with sustainability it appears e-mobility services has not gained particular momentum. Despite the strong Government support for EVs in Norway and the success of Tesla's amongst other models in Oslo, surprisingly there is not an overwhelming number of e-car clubs in the city [21]. It could be argued this is due to the numerous incentives and subsidies that make it attractive to own an EV. Although the policies set have been very positive for the EV market, it has not assisted in diminishing congestion but rather increased it. Consequently there is rising pressure on Norwegian ministers to reduce incentives for EVs. Alternatively, funding could be redirected to support schemes to reduce vehicle ownership such as e-mobility services [22].

\section{Discussion}

It is inevitable that urban mobility will adapt to regulatory pressures of climate change, resulting in gradual political and economic changes [23]. However, due 
to the stability of the transport sector the rate of which innovation can compete with the existing system is gradual [23]. Currently mobility services are acting in 'coalition' (albeit with small market share) to the dominant system of internal combustion owned vehicles. PSS requires a fundamental shift in culture, resources and behaviour in order to overcome the psychological barriers, whether it is in companies or the general populous.

Current cultural norms around car ownership and driving practices affect the uptake of e-mobility services. For instance it is reported that the "UK's strong culture and tradition of private vehicle ownership" were more significant than expected, one of the reasons for Car2Go exiting the market [24]. This has been shaped by the UK economy and tax regime but it has now become embedded within British culture. However younger generations (Generation Y and Z, from 1980 onwards) have begun to challenge this, largely due to the high cost of living, opening up the opportunity for more service based models.

PSSs diversify the market for customers, introducing new services, business models and vehicle technology. These will "simultaneously weaken the dominant position of the 'individual car' system, and support alternative transition pathways" [14]. The examples examined in Section 3 highlight four different business models with varying degrees of user flexibility, whilst offering alternatives to car ownership and fuel type. Having a wider portfolio of e-mobility services across car sharing, leasing and pooling will likely increase suitability to a wider populous and therefore adoption rate. Over time it will become evident which service has greatest demand, at which point market forces will respond and it will become a more competitive PSS.

As Mont [9] suggests there are three approaches of PSS that can be introduced (i) the sale of the use of the product instead of the product itself, (ii) the change to a leasing society, and (iii) the change in consumer attitudes from sales to service orientation. As it has been identified, e-mobility services require approaches (i) and (iii) in any given scenario and approach (ii) more specifically in product leasing applications. The extent to which these approaches are achieved indicates the rate and degree of which e-mobility will be adopted; a way of which to measure this is not currently considered but deserves further attention.

It is suggested by Brown et al. [25] that there are two scenarios regarding a collective change, either the innovation is widely adopted or the innovation is more slowly diffused through society until it reaches a critical mass. This aligns with the transition pathways strategy of socio-technical systems which can be categorised in to (i) the adaptation of a dominant system, when an innovation is widely adopted and becomes the dominant system through gradual changes supported by a coalition of actors; or (ii) the attempt to take over the dominant position which can create unstructured transition strategies and unaligned forces [14]. Currently e-mobility services are gradually entering the market along an established transition pathway due to societal forces and cultural norms that prevent quick diffusion, along with no direct policy incentive. Therefore the niche application of EVs requires greater support from policy in order to gain sufficient momentum for a socio-technical transition. 
Transitioning to a functional 'experience' economy shifting the role of the manufacturer to the provision of services is argued to be complimentary to the penetration of new products $[9,26]$. Furthermore customers are "willing to pay more than would be justified on the basis of "rational' calculation" due to intangible added value [10]. However as there is only a limited network that supports e-mobility, further investment in infrastructure (hardware and software) is required by city operators to make it a viable proposition. This is intensified as transitioning to e-mobility moves the point of profit from a product sale to the point of service that could be long term contracts or memberships. This requires "close integration of all actors within the life-cycle of a product-service" [9].

Marletto [14] argues there needs to be a multilevel and multidimensional policy for integrated urban transport by which EVs can play a secondary role. The difficulty is to approximate the necessary level of motivation crowding to influence users within reasonable margins. It is also necessary to consider regulations and other measures such as tax incentives to ensure e-mobility schemes are viable [27]. Car sharing and leasing are based on "the artefactual system remains more less the same, but where innovative institutional arrangements produce beneficial changes" [12]. Supporting policies incentivising car sharing for those in the life-cycle of the product/service will further encourage the transition. Additionally, policy makers could use e-mobility services as a strategy to target key groups i.e. those who suffer from transport poverty.

Mont [28] suggests companies follow a five step process to develop a PSS: (i) initial review of existing activities, (ii) marketing analysis, (iii) feasibility analysis, (iv) implementation, and (v) continuous system development. These should be in accordance to economic, social and environmental sustainability criteria determined by the company. This should be applicable when transitioning to e-mobility services from a city perspective (working in partnership with infrastructure and service providers); examining the city platform to see where and what services can be used. This methodology "identifies critical success factors, such as consumer perception of trust in the function provider, constant feedback concerning system function, changing customer needs (and) necessary changes in the system" [29]. This requires regular stakeholder consultation to monitor efficiencies and user satisfaction from the perspective of a service user and city inhabitant.

Finally, as previously identified two (of the five) of Ehrenfeld's [12] evaluative criteria: (i) changes in vehicle ownership structure and (ii) changes in modes of producer-user interactions, are key in considering transitioning to e-mobility services. The examples considered in this paper have demonstrated a market for alternative ownership structures and producer-user relationships that have proven to be successful in the urban context. In order to fully optimise e-mobility services, alongside public transport, real-time data of vehicle/charging availability should be accessible to users. 


\section{Conclusions}

E-mobility services can substantially decrease a city's transport emissions and congestion through reduced car ownership and car mileage. This requires transitioning to a PSS approach with new market concepts and business models. This paper has explored various use-oriented models to understand how cities can introduce MaaS.

Adopting PSSs can introduce new revenue streams for companies in new market segments and can facilitate innovation and competitiveness [9]. This is particularly so in a mature industry such as the automotive industry. BMW for example, have introduced a service to their standard portfolio (DriveNow) at a high level of quality that will be difficult to replicate. Although this remains a niche market and market offer it introduces a new approach to travel. Useorientated services challenge the embedded path dependent characteristics and social norms of car ownership, fuel type and revenue structure.

The e-mobility services explored highlight the need for society to have a new relationship with the car, adapting to a PSS requires a significant change in behaviour. The premise of PSSs to continuously innovate means the market will be led by a combination of market and societal forces. As the market develops and more players compete, society will determine the dominant use-oriented service. Until then, e-mobility services are being diffused through adaption of the dominant ICE asset system.

Taking a combined approach to city transport can reduce congestion, air pollution and emissions but requires an open data platform to be accessible to monitor availability. In order not to put excess pressure on existing city services, especially during peak demand, governments should work closely with service providers. Furthermore target audiences or geographical areas could be focused upon with the use of tax incentives and grants.

Further research will conduct interviews with service providers, such as DriveNow. These will be semi-structured interviews across the three use-oriented services to elaborate on the existing knowledge of PSSs and applicable transition pathways within the transport sector. Additional research should be conducted on the environmental implications of e-mobility services, considering the vehicle lifecycle and the substituted use of owned vehicles.

\section{References}

[1] KPMG, "KPMG's Gobal Automotive Executive Survey 2013”, 2013. (Online). Available: http:/www.kpmg.com/RU/en/IssuesAndInsights/ ArticlesPublications/Documents/KPMGs-Global-Automotive-ExecutiveSurvey-2013.pdf. (Accessed 22 February 2013).

[2] European Commision, "Reducing $\mathrm{CO}_{2}$ emissions from passenger cars", 2015. (Online). Available: http://ec.europa.eu/clima/policies/transport/ vehicles/cars/index_en.htm. (Accessed 17 March 2015).

[3] OLEV, "Making the Connection. The Plug-In Vehicle Infrastructure Strategy", Department for Transport, London, 2011. 
[4] Geels, "Processes and patterns in transitions and system innovations: Refining the co-evolutionary multi-level perspective", Technological Forecasting and Social Change, vol. 72, no. 6, pp. 681-696, 2005.

[5] LSE Cities, "Going Green. How cities are leading the next economy", London School of Economics and Political Science, London, 2013.

[6] Weiller, "E-Mobility Services. New economic models for transport in the digital economy", University of Cambridge, Service Alliance, Cambridge, 2012.

[7] Wells and Nieuwenhuis, "The automotive industry - a guide", Centre for Automotive Industry Research (CAIR), Cardiff and British Telecommunications plc, London, 2001.

[8] Goedkoop, Halen, Riele and Rommens, "Product service system ecological and economic basis", Price Waterhouse Coopers/Pi!MC, Storm CS, 1999.

[9] Mont, "Clarifying the concept of product-service system", Cleaner Production, vol. 10, no. 3, pp. 237-245, 2002.

[10] Tukker, "Eight Types of Product-Service System: Eight Ways to Sustainability? Experiences from Suspronet", Business Strategy and the Environment, vol. 13, pp. 246-260, 2004.

[11] Williams, "Productservice system in the automobile industry: contribution to system innovation?" Cleaner Production, vol. 15, pp. 1093-1103, 2007.

[12] Ehrenfeld, "Designing 'sustainable' product/serive systems," in Ecodesign, Tokyo, 2001.

[13] BMW, “ $360^{\circ}$ Electric”, BMW, 2015. (Online). Available: http://www.bmw.com/com/en/insights/corporation/bmwi/ 360_electric.html. (Accessed 18 March 2015).

[14] Marletto, "Car and the city: Socio-technical transition pathways to 2030", Technological Forecasting \& Social Change, vol. 87, pp. 164-178, 2014.

[15] Autolib', "Our Commitment". (Online). Available: https://www.autolib.eu/ en/our-commitment/urban-revolution/. (Accessed 24 September 2014).

[16] Holland, "Autolib' Electric Carsharing Program". (Online). Available: http://cleantechnica.com/2014/04/02/autolib-electric-carsharing-program/. (Accessed 29 September 2014).

[17] Henley, "Electric 'Boris cars' are coming to London - how do they work in Paris?" (Online). Available: http://www.theguardian.com/cities/2014/jul/ 09/electric-boris-car-source-london-how-work-paris-autolib. (Accessed 24 September 2014).

[18] Claris, "The future of urban mobility", 16 March 2015. (Online). Available: http://yourbetterbusiness.co.uk/the-future-of-urban-mobility-susan-claris/. (Accessed 16 March 2015).

[19] Seal-Driver, "Flexible car sharing from BMWi, Mini and Sixt". (Online). Available: www.carplus.org.uk/wp.../J-Seal-Driver-Drive-Nowpresentation.pdf. (Accessed 20 September 2014).

[20] Hertz, "BilPool". (Online). Available: http://www.hertzbilpool.no/. (Accessed 25 September 2014). 
[21] Vidal, "Norway has fallen in love with electric cars - but the affair is coming to an end". (Online). Available: http://www.theguardian.com/environment/ 2014/jan/29/norway-electric-cars-sale. (Accessed 20 September 2014).

[22] Wirgman, "Norwegian Govt under increasing pressure to reduce incentives for EVs". (Online). Available: https://www.zap-map.com/norwegian-govtincreasing-pressure-reduce-incentives-evs/\#.VCu6UMJdW0M. (Accessed 20 September 2014).

[23] Geels, "From sectoral systems of innovation to socio-technical systems: Insights about dynamic and change from sociology and institutional theory", Research Policy, vol. 33, pp. 897-920, 2004.

[24] BBC, "Car-sharing scheme car2go to withdraw from the UK". (Online). Available: http://www.bbc.com/news/uk-england-birmingham-27546644. (Accessed 29 September 2014).

[25] Brown, Vergragt, Green and Berchicci, "Learning for sustainability transition through bounded socio-technical experiments in personal mobility", Technology Analysis and Strategic Management, vol. 15, no. 3, pp. 291-315, 2003.

[26] Fioruzzi, "Dematerialization and service economy: how good it is?" 1997. (Online). Available: http://www.cyen.com/green_value/paper1.htm. (Accessed 19 March 2015).

[27] Roy, "Sustainable product-service systems", Futures, vol. 32, pp. 289-299, 2000.

[28] Mont, "Introducing and developing a product-service system (PSS) concept in Sweden", International Institute for Industrial Environmental Economics (IIEE) Report, Lund University, 2001.

[29] Williams, "Product-service systems in the automotive industry: the case of micro-factory retailing", Journal of Cleaner Production, vol. 14, pp. 172184, 2006.

[30] Jackson, Material concerns. Pollution, profit and the quality of life, London: Routledge, 1996. 